\title{
A northern sky survey for ultra-high-energy gamma-ray source using the Tibet air-shower array and muon-detector array.
}

\section{Xu Chen ${ }^{a, *}$ on behalf of the Tibet AS $\gamma$ Collaboration}

(a complete list of authors can be found at the end of the proceedings)

${ }^{a}$ Key Laboratory of Particle Astrophysics, Institute of High Energy Physics, Chinese Academy of Sciences, Beijing 100049, China

E-mail: chenxu@ihep.ac.cn

The Tibet AS $\gamma$ experiment located at $4300 \mathrm{~m}$ above sea level, Tibet, China, has a wide field of view and large effective area. It consists of the Tibet air-shower array (Tibet-AS), the air-shower coredetector array (YAC) and the underground water-Cherenkov muon-detector array (Tibet-MD). The Tibet-MD array significantly improves $\gamma$-ray sensitivity in the $10-1000 \mathrm{TeV}$ energy region by an order of magnitude better than any other previously existing experiments in the world. In this work we will present the catalog of TeV $\gamma$-ray sources using 719 days of data from the Tibet AS $\gamma$ experiment. The catalog represents the most sensitive survey of the northern $\gamma$-ray sky at energies above several tens of TeV. These ultra-high-energy $\gamma$-ray sources are believed to be related to pulsars and supernova remnants.

$37^{\text {th }}$ International Cosmic Ray Conference (ICRC 2021)

July 12th-23rd, 2021

Online - Berlin, Germany

\footnotetext{
${ }^{*}$ Presenter
} 


\section{Introduction}

The all-sky survey remains one of the major concerns for Extensive Air Shower experiments, as they have a wide field of view and a high duty cycle. The Tibet AS $\gamma$ experiment, have observed gamma-ray emissions from Crab, Mrk501, Mrk421 [1-3]. Since 2014, the underground waterCherenkov moun detetor(MD) array with a total area of $3400 \mathrm{~m}^{2}$ started operation. We can effectively discriminate between primary gamma rays and cosmic-ray backgrounds by means of counting the number of muons in an air shower event, because a gamma-ray induced air shower has significantly less muons than a cosmic-ray induced one. Thus the gamma-ray sensitivity is significantly improved in the $10-1000 \mathrm{TeV}$ energy region.

We first detect the highest energy photons beyond $100 \mathrm{TeV}$ from an astrophysical source, the standard candle Crab nebula[4], and its broad-band spectra can be explained dominated by leptonic origin via the inverse Compton scattering by relativistic electrons[5]. We also detect veryhigh-energy gamma-ray emission around the direction of SNR G106.3+2.7, and the morphological feature of these gamma rays appears to favour a hadronic origin via the $\pi^{0}$ decay caused by accelerated relativistic protons over a leptonic origin[6]. By analysising sub-PeV diffuse gamma rays destribution, We found the gamma ray distribution is extended around the Galactic plane. This is strong evidence for ubiquitous Galactic cosmic rays beyond PeV[7].

\section{The Tibet AS $\gamma$ Experiment}

The new Tibet hybrid extensive air shower experiment has been operated at Yangbajing (E $90^{\circ} 31^{\prime}, \mathrm{N} 30^{\circ} 6^{\prime}, 4300 \mathrm{~m}$ above sea level) in Tibet, China, and data taking started from 2014. This hybrid experiment currently consists of three types of detector array, including the Tibet AS array (Tibet-AS), an underground water-Cherenkov muon-detector array (MD) and the Yangbajing AS core-detector array (YAC-II). These systems are used to observe air showers of high energy celestial gamma-ray origin and those of nuclear-component origin. The currently configuration of the Tibet $\mathrm{AS}+\mathrm{MD}$ arrays are shown in Fig.1.

The Tibet-AS array consists of 597 plastic scintillation detectors, each with an area of 0.5 $\mathrm{m}^{2}$, totally covering an area of $65700 \mathrm{~m}^{2}$ with an inner area of $50400 \mathrm{~m}^{2}$ indicated by dashed-dot line and dashed line. This array is used to detect the densities and arriving times of partcles in air-shower events, and then these infomations are used to reconstruct the direction and energy of primary particles. The angle and energy resolutions of primary particles can be estimated to be approximately $0.5^{\circ}$ and $40 \%$ at $10 \mathrm{TeV}, 0.2^{\circ}$ and $20 \%$ at $100 \mathrm{TeV}$ for gamma rays[4].

The MD array consists of 4 pools, with 16 cells for each. Each cell has an area of $7.35 \mathrm{~m}$ $\times 7.35 \mathrm{~m}$. These cell are set up underground and filled with water with $1.5 \mathrm{~m}$ depth. Muons in excess of $1 \mathrm{GeV}$ associated with air showers are observed by detecting Cherenkov lights with 20-inch-diameter photomultiplier tubes(PMTs) mounted downward on the ceiling of each pool, while most of the electromagnetic components such as electrons, positrons and photons are blocked by the soil with depth of $2.4 \mathrm{~m}$. Utilizing the fact that gamma-ray-induced air showers contain fewer muons compared with cosmic-ray-induced ones we can discriminate photon signals from cosmic-ray backgrounds by means of counting muon number detected by the MD array. Thus, the 


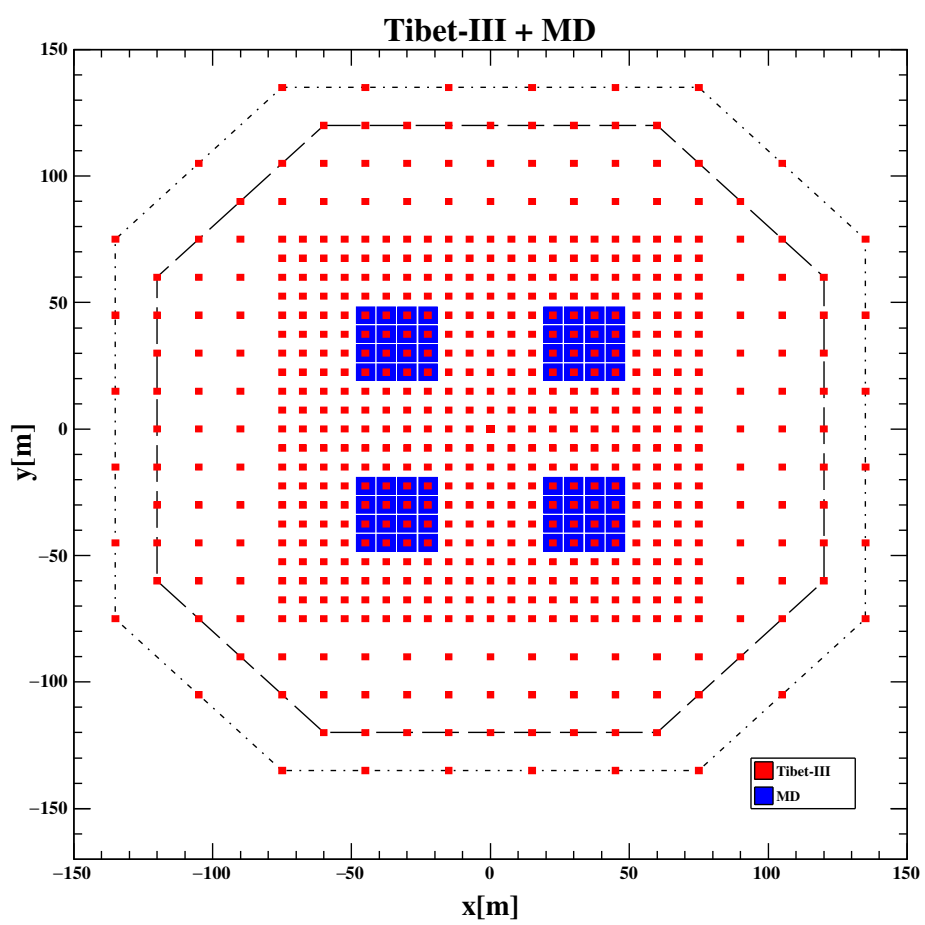

Figure 1: Schematic view of the Tibet-AS+MD array. Red and blue filled squares denote Tibet-AS scintillation detectors and MD muon detectors. The area enclosed by the dashed-dot line indicates the total $65700 \mathrm{~m}^{2}$ area. The area enclosed by the dashed line indicates the inner $50400 \mathrm{~m}^{2}$ area of AS array.

MD array significantly improve the sensitivity of the Tibet AS array towards gamma-ray signals above $10 \mathrm{TeV}$.

\section{Analysis}

In this work, we use data obtained by both Tibet-AS and MD with 719 live days from 2014 February to 2017 May. The direction of primary particle was reconstructed using the same way as in the past[8]. The energy of primary particle was reconstructed using the lateral distribution of the density of secondary particles $(\rho)$, we use the density at distance of $50 \mathrm{~m}$ from the air-shower core as a better energy estimator[9]. We use the number of all muon particles $\left(\sum \mu\right)$ as the parameter for $\mathrm{P} / \gamma$ separation. The gamma-like events should be muon-poor, and the energy-dependent muon-cut condition is the same as used in previous works[4].

The event selection was done by imposing the following criteria: (1) Each shower event should fire four or more Tibet-AS detectors recording more than 1.25 particles. (2) Thee number of available detectors for the AS reconstruction is larger than 16 (3) The estimated shower center location should be inside the array. (4) The energy larger than $10 \mathrm{TeV}$. (5) The zenith angle of the incident direction should be less than $60^{\circ}$. (6) The gamma-like events.

The skymap is divided base on HEALPIX framework[10] with order=10, each of which has an approximate size of $0.05^{\circ} \times 0.05^{\circ}$ we adopt the equizenith angle method to estimate the background 
of cosmic rays for each pix as used in previous works[3, 4, 8, 11], The number of cosmic-ray background events is estimated from the number of events averaged over 10 off-source windows located at the same zenith angle with the on-source window. The windows size is set according to different situations.

\section{Results}

Fig. 2 show the northern survey using the point source searching mode(with small size of searching windows depends on the energy). We totally find 12 gamma-ray sources with five-sigma significance, all of these gamma-ray sources are along the galaxy plane, including the brighest $\mathrm{TeV}$ $\gamma$-ray source standard candle.

Table. 1 lists these gamma-ray sources, and its associated sources on TeVCat[12]. All of these regions have at least one $\mathrm{TeV}-\gamma$-ray source within $0.5^{\circ}$ distance, except one $\left(R A=279.91^{\circ}, D E=\right.$ $\left.-6.03^{\circ}\right)$. There are two extended undefined source HESS J1841-055, HESS 1837-069 nearby[13], it can be the principal maximum of an elongated region containing multiple known extended sources.

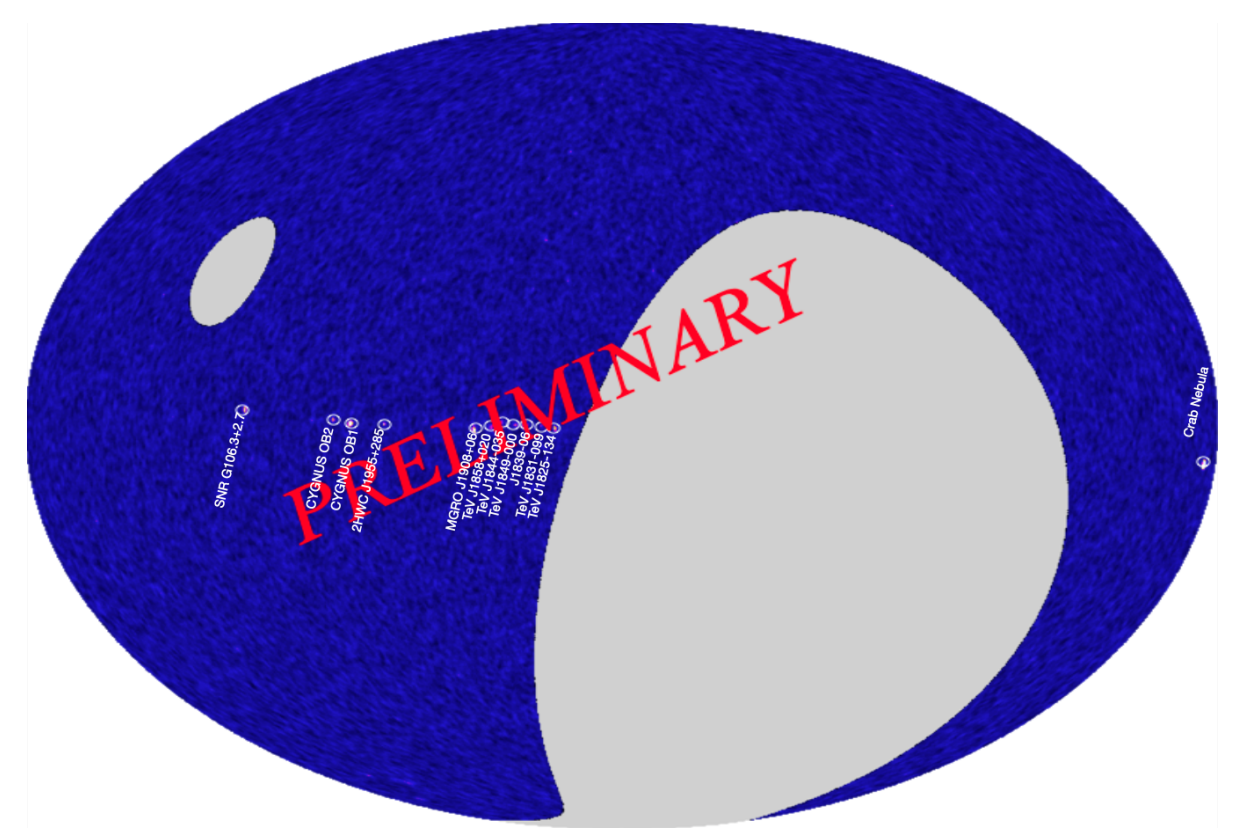

Figure 2: Sigificance map of the Tibet-AS+MD northern sky gamma-ray survey using the galactic coordinate system. The white circle indicated the direction of excess with sigificance $>5$ above $10 \mathrm{TeV}$. The grep region is outside of the field of view.

For large extend $\gamma$-ray halos, there is no significance using the same method as previous. We use large smoothing windows and "the Equi-Declination Method". Fig.3 show the sigificance map around the geminga region above $10 \mathrm{TeV}$, by using $3^{\circ}$ smoothing window. We can see the excess emmision around the geminga pulsar, and there is also a excess around the south-east PSR B0656+14. 


$\begin{array}{lllll}\text { Associated sources } & \mathrm{Ra}[\mathrm{deg}] & \mathrm{De}[\mathrm{deg}] & \mathrm{b}[\mathrm{deg}] & \mathrm{D} \\ \text { Crab } & 83.65 & 22.02 & -5.76 & 0.0 \\ \text { TeV J1825-134 } & 276.52 & -13.4 & -0.58 & 0.05 \\ \text { TeV J1831-099 } & 277.58 & -9.84 & 0.16 & 0.27 \\ \text { TeV J1837-065 } & 279.91 & -6.03 & -0.14 & 0.70 \\ \text { TeV J1840-055 } & & & & 0.63 \\ \text { TeV J1844-035 } & 280.92 & -3.85 & -0.03 & 0.26 \\ \text { TeV J1849-000 } & 282.84 & 0.03 & -0.00 & 0.45 \\ \text { TeV J1857+026 } & 284.70 & 2.66 & -0.42 & 0.40 \\ \text { MGRO J1908+06 } & 287.01 & 6.20 & -0.85 & 0.07 \\ \text { 2HWC J1955+285 } & 298.87 & 28.63 & 0.17 & 0.05 \\ \text { Cygnus OB1 } & 305.02 & 36.77 & 0.23 & 0.23 \\ \text { Cygnus OB2 } & 308.01 & 41.19 & 0.89 & 0.13 \\ \text { SNR G106.3+2.7 } & 336.77 & 60.88 & 2.77 & 0.11\end{array}$

Table 1: Result by the Tibet-AS+MD for sigificant region above $10 \mathrm{TeV}$ and its associated gamma-ray sources in TeVCat. Ra, De are the positions on $\mathrm{J} 2000$ coordinate system, and b is the Galactic latitude. D represent the angle distance from the TeVCat sources to the Tibet-AS+MD sigificant area.

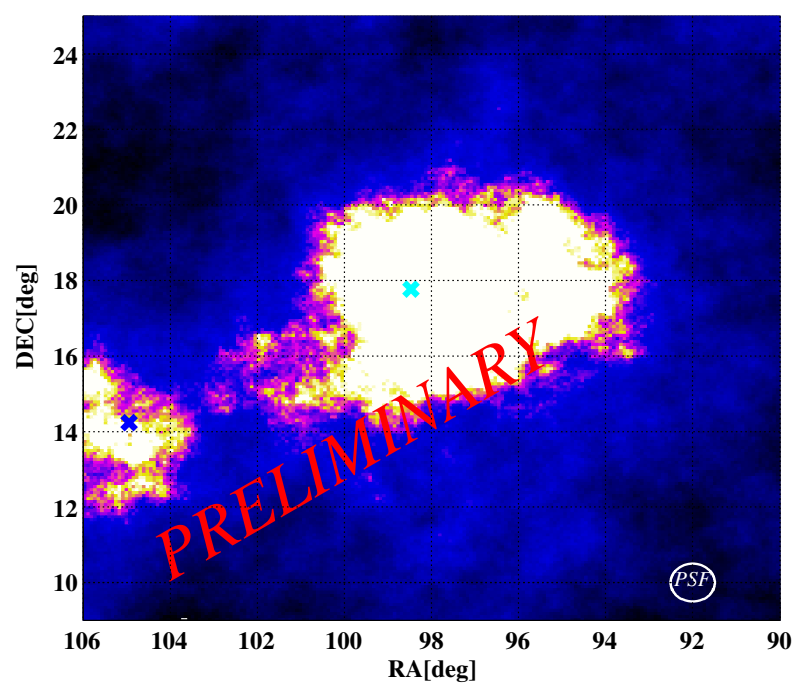

Figure 3: Sigificance map of the $\gamma$-ray emmision around Geminga. The cyan cross $\mathrm{X}$ indicate the position geminga pulsar, and the blue cross $\mathrm{X}$ indicate the position of PSR B0656+14. The white circle at the right-bottom indicate the angle resolution of the Tibet-AS array.

\section{Conclusions}

In this paper, we report 12 gamma-ray sources above $10 \mathrm{TeV}$. All of these gamma-ray sources are along the galaxy plane. We also shows the large extended gamma-ray emission around the geminga region. These results show the strength of the underground muon detectors for discriminating 
primary gamma-ray from cosmic-ray backgounds.

\section{Acknowledgements}

The collaborative experiment of the Tibet Air Shower Arrays has been conducted under the auspices of the Ministry of Science and Technology of China and the Ministry of Foreign Affairs of Japan. This work was supported in part by a Grant-in-Aid for Scientific Research on Priority Areas from the Ministry of Education, Culture, Sports, Science and Technology, and by Grants-in-Aid for Science Research from the Japan Society for the Promotion of Science in Japan. This work is supported by the National Key R\&D Program of China (No. 2016YFE0125500), the Grants from the National Natural Science Foundation of China (Nos. 12073050, 11533007, 11673041, $11873065,11773019,11773014,11633007,11803011$ and 11851305), and the Key Laboratory of Particle Astrophysics, Institute of High Energy Physics, CAS. This work is also supported by the joint research program of the Institute for Cosmic Ray Research (ICRR), the University of Tokyo.

\section{References}

[1] M. Amenomori et al.apjl, 525 (1999) L93.

[2] M. Amenomori et al.apj, 532 (2000) 302.

[3] M. Amenomori et al.apj, 598 (2003) 242.

[4] M. Amenomori et al.prl, 123 (2019) 051101.

[5] X. Zhang et al.mnras, 497 (2020) 3477.

[6] T.A. Collaboration et al.Nature Astronomy, 5 (2021) 460.

[7] M. Amenomori et al.prl, 126 (2021) 141101.

[8] M. Amenomori et al.apj, 692 (2009) 61.

[9] K. Kawata et al.Experimental Astronomy, 44 (2017) 1.

[10] K.M. Górski et al.apj, 622 (2005) 759.

[11] M. Amenomori et al.apj, 633 (2005) 1005.

[12] S.P. Wakely et al. in International Cosmic Ray Conference, vol. 3 of International Cosmic Ray Conference, pp. 1341-1344, Jan., 2008.

[13] H.E.S.S. Collaboration et al.aap, 612 (2018) A1. 


\section{Full Authors List: Tibet AS $\gamma$ Collaboration}

M. Amenomori ${ }^{1}$, S. Asano ${ }^{2}$, Y. W. Bao ${ }^{3}$, X. J. Bi ${ }^{4}$, D. Chen ${ }^{5}$, T. L. Chen ${ }^{6}$, W. Y. Chen ${ }^{4}, \mathrm{Xu} \mathrm{Chen}^{4}$, Y. Chen ${ }^{3}, \mathrm{Cirennima}^{6}$, S. W. Cui ${ }^{7}$, Danzengluobu $^{6}$, L. K. Ding ${ }^{4}$, J. H. Fang ${ }^{4,8}$, K. Fang ${ }^{4}$, C. F. Feng ${ }^{9}$, Zhaoyang Feng ${ }^{4}$, Z. Y. Feng ${ }^{10}$, Qi Gao ${ }^{6}$, A. Gomi ${ }^{11}$, Q. B. Gou ${ }^{4}$, Y. Q. Guo ${ }^{4}$, Y. Y. Guo ${ }^{4}$, H. H. He ${ }^{4}$, Z. T. He ${ }^{7}$, K. Hibino ${ }^{12}$, N. Hotta ${ }^{13}$, Haibing Hu${ }^{6}$, H. B. Hu ${ }^{4}$, K. Y. Hu $u^{4,8}$, J. Huang ${ }^{4}$, H. Y. Jia ${ }^{10}$, L. Jiang ${ }^{4}$, P. Jiang ${ }^{5}$, H. B. Jin ${ }^{5}$, K. Kasahara ${ }^{14}$, Y. Katayose ${ }^{11}$, C. Kato ${ }^{2}$, S. Kato ${ }^{15}$, T. Kawashima ${ }^{15}$, K. Kawata ${ }^{15}$, M. Kozai ${ }^{16}$, D. Kurashige ${ }^{11}$, Labaciren ${ }^{6}$, G. M. Le ${ }^{17}$, A. F. Li ${ }^{18,9,4}$, H. J. Li ${ }^{6}$, W. J. Li ${ }^{4,10}$, Y. Li ${ }^{5}$, Y. H. Lin ${ }^{4,8}$, B. Liu ${ }^{19}$, C. Liu ${ }^{4}$, J. S. Liu ${ }^{4}$,

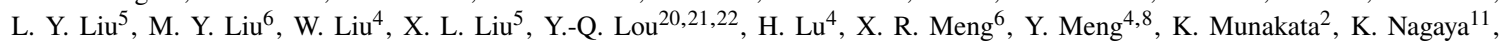
Y. Nakamura ${ }^{15}$, Y. Nakazawa ${ }^{23}$, H. Nanjo ${ }^{1}$, C. C. $\mathrm{Ning}^{6}$, M. Nishizawa ${ }^{24}$, M. Ohnishi ${ }^{15}$, S. Okukawa ${ }^{11}$, S. Ozawa ${ }^{25}$, L. Qian $^{5}$, X. Qian ${ }^{5}$, X. L. Qian $^{26}$, X. B. Qu ${ }^{27}$, T. Saito ${ }^{28}$, Y. Sakakibara ${ }^{11}$, M. Sakata ${ }^{29}$, T. Sako ${ }^{15}$, T. K. Sako ${ }^{15}$, J. Shao ${ }^{4,9}$, M. Shibata ${ }^{11}$, A. Shiomi ${ }^{23}$, H. Sugimoto ${ }^{30}$, W. Takano ${ }^{12}$, M. Takita ${ }^{15}$, Y. H. Tan ${ }^{4}$, N. Tateyama ${ }^{12}$, S. Torii ${ }^{31}$, H. Tsuchiya $^{32}$, S. Udo $^{12}$, H. Wang $^{4}$, Y. P. Wang ${ }^{6}$, Wangdui ${ }^{6}$, H. R. $\mathrm{Wu}^{4}$, Q. $\mathrm{Wu}^{6}$, J. L. $\mathrm{Xu}^{5}$, L. Xue ${ }^{9}$, Z. Yang ${ }^{4}$, Y. Q. Yao ${ }^{5}$, J. Yin ${ }^{5}$, Y. Yokoe ${ }^{15}$, N. P. Yu ${ }^{5}$, A. F. Yuan ${ }^{6}$, L. M. Zhai ${ }^{5}$, C. P. Zhang ${ }^{5}$, H. M. Zhang ${ }^{4}$, J. L. Zhang ${ }^{4}$, X. Zhang ${ }^{3}$, X. Y. Zhang ${ }^{9}$, Y. Zhang ${ }^{4}$, Yi Zhang ${ }^{33}$, Ying Zhang ${ }^{4}$, S. P. Zhao ${ }^{4}$, Zhaxisangzhu $^{6}$ and X. X. Zhou ${ }^{10}$

\footnotetext{
${ }^{1}$ Department of Physics, Hirosaki University, Hirosaki 036-8561, Japan.

${ }^{2}$ Department of Physics, Shinshu University, Matsumoto 390-8621, Japan.

${ }^{3}$ School of Astronomy and Space Science, Nanjing University, Nanjing 210093, China.

${ }^{4}$ Key Laboratory of Particle Astrophysics, Institute of High Energy Physics,

Chinese Academy of Sciences, Beijing 100049, China.

${ }^{5}$ National Astronomical Observatories, Chinese Academy of Sciences, Beijing 100012, China.

${ }^{6}$ Department of Mathematics and Physics, Tibet University, Lhasa 850000, China.

${ }^{7}$ Department of Physics, Hebei Normal University, Shijiazhuang 050016, China.

${ }^{8}$ University of Chinese Academy of Sciences, Beijing 100049, China.

${ }^{9}$ Institute of Frontier and Interdisciplinary Science and Key Laboratory of Particle Physics and Particle Irradiation (MOE), Shandong University, Qingdao 266237, China.

${ }^{10}$ Institute of Modern Physics, SouthWest Jiaotong University, Chengdu 610031, China.

${ }^{11}$ Faculty of Engineering, Yokohama National University, Yokohama 240-8501, Japan.

${ }^{12}$ Faculty of Engineering, Kanagawa University, Yokohama 221-8686, Japan.

${ }^{13}$ Faculty of Education, Utsunomiya University, Utsunomiya 321-8505, Japan.

${ }^{14}$ Faculty of Systems Engineering, Shibaura Institute of Technology, Omiya 330-8570, Japan.

${ }^{15}$ Institute for Cosmic Ray Research, University of Tokyo, Kashiwa 277-8582, Japan.

${ }^{16}$ Institute of Space and Astronautical Science, Japan Aerospace Exploration Agency (ISAS/JAXA), Sagamihara 252-5210, Japan.

${ }^{17}$ National Center for Space Weather, China Meteorological Administration, Beijing 100081, China.

${ }^{18}$ School of Information Science and Engineering, Shandong Agriculture University, Taian 271018, China.

${ }^{19}$ Department of Astronomy, School of Physical Sciences, University of Science and Technology of China, Hefei 230026, China.

${ }^{20}$ Department of Physics and Tsinghua Centre for Astrophysics (THCA), Tsinghua University, Beijing 100084, China.

${ }^{21}$ Tsinghua University-National Astronomical Observatories of China (NAOC) Joint Research Center for Astrophysics, Tsinghua University, Beijing 100084, China.

${ }^{22}$ Department of Astronomy, Tsinghua University, Beijing 100084, China.

${ }^{23}$ College of Industrial Technology, Nihon University, Narashino 275-8576, Japan.

${ }^{24}$ National Institute of Informatics, Tokyo 101-8430, Japan.

${ }^{25}$ National Institute of Information and Communications Technology, Tokyo 184-8795, Japan.

${ }^{26}$ Department of Mechanical and Electrical Engineering,

Shangdong Management University, Jinan 250357, China.

${ }^{27}$ College of Science, China University of Petroleum, Qingdao 266555, China.

${ }^{28}$ Tokyo Metropolitan College of Industrial Technology, Tokyo 116-8523, Japan.

${ }^{29}$ Department of Physics, Konan University, Kobe 658-8501, Japan.

${ }^{30}$ Shonan Institute of Technology, Fujisawa 251-8511, Japan.

${ }^{31}$ Research Institute for Science and Engineering, Waseda University, Tokyo 162-0044, Japan.

${ }^{32}$ Japan Atomic Energy Agency, Tokai-mura 319-1195, Japan.

${ }^{33}$ Key Laboratory of Dark Matter and Space Astronomy, Purple Mountain Observatory,

Chinese Academy of Sciences, Nanjing 210034, China.
} 